Rev. Elev. Méd. vét. Pays trop., 1974, 27 (2) : 195-201

\title{
La distomatose du lapin domestique à Fasciola gigantica
}

\author{
par M. GRABER (*) \\ (avec la collaboration technique de Mme J. GEVREY)
}

\begin{abstract}
RESUME
Douze lapins français reçoivent chacun 30 à 200 Métacercaires de Fasciola gigantica de 18,64 et 158 jours, conservées à $+4^{\circ} \mathrm{C}$.

Trois animaux réagissent positivement à l'infestation expérimentale, mais une seule Douve a été recueillie dans le parenchyme hépatique.

L'auteur compare ces résultats à ceux obtenus dans d'autres pays. Il constate que le lapin réagit irrégulièrement à l'infestation par Fasciola gigantica et que sa réceptivité varie en fonction des souches utilisées et du nombre de Métacercaires administrées.
\end{abstract}

On sait que le lapin domestique, Oryctolagus cuniculus Linné, est sensible à l'infestation par Fasciola hepatica et qu'il est relativement aisé, chez ce rongeur, de reproduire la distomatose hépato-biliaire, ce qui en facilite l'étude.

Aussi, cette aptitude a-t-elle été mise à profit, dès la fin du siècle dernier, dans divers laboratoires d'Europe, d'Amérique et d'Asie dont les travaux ont été axés principalement sur les conditions d'infestation, l'étude clinique et anatomique, la pathogénie et l'immunologie de la maladie, ainsi que sur la pathologie comparée de Fasciola hepatica et de Fasciola gigantica (10). En outre, des essais thérapeutiques, destinés à préciser le pouvoir antidistomien de certains médicaments, ont été effectués sur des lapins artificiellement infestés.

Les résultats obtenus ont donné lieu à de nombreuses publications dont il est impossible ici de donner la liste complète. Tout au plus, peut-on citer, parmi les plus connues, celles de KIMURA $(23,22)$, d'URQUARTH (32, 33), de GIGITASHIVILI (14), de DARGIE et Collab. $(7,8,9)$, d'HOLMES et Collab. (18),

(*) Ecole Nationale Vétérinaire de Lyon, Chaire de Parasitologie, 2, quai Chauveau, 69337 Lyon Cedex 1. de GOLDBERGIENNE (15), de MOVSESIJAN et Collab. (25), de KENDALL et Collab. (21) et de ROSS (27).

En revanche, dans les pays tropicaux où Fasciola gigantica est l'espèce dominante, les chercheurs ont surtout utilisé, comme animaux d'expérience, des ruminants domestiques, c'està-dire des zébus, des bœufs, des moutons et des chèvres.

Malheureusement, pour des raisons diverses, il n'est pas toujours possible d'opérer ainsi et il a paru utile de rechercher dans quelle mesure le lapin qui est également réceptif à Fasciola gigantica (4) pouvait se substituer à ces espèces. C'est l'objet du présent travail qui a été réalisé à l'Ecole Nationale Vétérinaire de Lyon (Chaire de parasitologie) au cours des années 1970 et 1971.

\section{MATERIEL ET METHODE}

\section{MATERIEL}

\subsection{Les éléments infestants}

Des Limnées, Limnaea natalensis, élevées au laboratoire dans un bac d'une dizaine de litres, 
ont été mises en contact le 13 février 1970 avec des œufs de Fasciola gigantica provenant de la vésicule biliaire d'un zébu atteint de distomatose et sacrifié à l'abattoir de N'djamena (République du Tchad).

L'infestation des Limnées a été contrôlée, de 15 jours en 15 jours, par écrasement de quelques exemplaires entre lame et lamelle. 52 jours plus tard, elles commencent à émettre des Cercaires. Elles sont alors placées dans une cuve renfermant de l'eau froide et cette opération est répétée plusieurs fois à une semaine d'intervalle.

Dans le récipient, sont disposés des morceaux de papier sur lesquels les Cercaires viennent s'enkyster.

Les métacercaires ainsi formées sont laissées une dizaine de jours à la température du laboratoire (phase de maturation), puis stockées dans de l'eau à $+4^{\circ} \mathrm{C}$.

On sait - tout au moins pour Fasciola hepatica - que cette température est favorable à leur conservation et que leur survie peut atteindre un an. Il semble en être de même pour Fasciola gigantica $(4,11)$ : au bout de 3 mois, 80 p. 100 des Métacercaires soumises à ce traitement sont capables de se désenkyster dans le milieu artificiel de Wikerhauser (3).

Au cours de cette expérience, la viabilité des Métacercaires a été testée à intervalles réguliers :

- soit par examen direct au microscope qui permet de mettre en évidence les «glandes de pénétration " (11) ou "granulations excrétrices $\gg(31)$ caractéristiques (photos 1 et 2) qui disparaissent, lorsque le kyste métacercarien est mort: dans ce cas, il se présente comme une masse diffuse, sans aucune structure.

- soit par chauffage à $37^{\circ} \mathrm{C}$, ce qui active les Métacercaires et leurs mouvements sont facilement observés à la loupe.

\subsection{Les animaux}

12 lapins, âgés de 4 à 5 mois et originaires d'une élevage de la région lyonnaise, ont été utilisés. Des examens coproscopiques effectués au moment de l'achat ont montré qu'aucun d'entre eux n'était porteur de parasites internes.

\section{METHODE}

Trois lots de quatre animaux chacun ont été constitués :

- le premier a reçu de 30 à 50 Métacercaires de 18 jours;

- le second 30 à 60 Métacercaires de 64 jours;

- le troisième 200 Métacercaires de 158 jours.

Après plus de cinq mois de réfrigérateur et, bien que les tests de viabilité aient été satisfaisants, il a paru utile, pour plus de sécurité, d'augmenter le nombre d'éléments infestants.

Les lapins ont été isolés dans des cages séparées. Les Métacercaires ont été soigneusement triées et, toutes celles qui paraissaient douteuses, éliminées. Elles ont été enveloppées dans des feuilles de salades que l'animal, préalablement mis à la diète, consomme assez facilement.

Après l'infestation, tous les quinze jours, il a été procédé à la recherche systématique des œufs de Fasciola gigantica dans les selles (sauf sur le lot $n^{\circ}$ III). La méthode employée est celle de Janeckso et Urbanek, d'enrichissement par flottaison en solution d'iodo-mercurate de potassium.

Les lapins ont été sacrifiés 57 à 101 jours après l'infestation initiale (tableau $n^{\circ} 1$ ).

Les foies ont fait l'objet d'un examen attentif à la loupe et, chaque fois que des lésions ont été décelées, elles ont aussitôt été prélevées pour études histologiques.

Les Douves, de leur côté, ont été extraites par la Méthode de Yalcin (34) : elle consiste à écraser à la main, dans un seau plein d'eau, le foie suspect. Le sang est éliminé par lavages successifs. On laisse déposer et on siphonne le liquide surnageant. On ajoute alors de l'eau enrichie d'eau oxygénée, à raison de 25 p. 100. 'Les particules de foie qui remontent à la surface sont retirées à l'aide d'une écumoire. Les Distomes demeurent au fond du seau et sont récoltés sur un plateau à fond noir.

\section{RESULTATS}

Ils figurent au tableau $\mathrm{n}^{\circ} \mathrm{I}$.

Sur les douze lapins mis en expérience, trois d'entre eux seulement présentent des lésions 
TABLEAU $\mathrm{N}^{\circ} \mathrm{I}$

Infestations des lapins par Fosciota gigantica

\begin{tabular}{|c|c|c|c|c|c|}
\hline \multirow{2}{*}{$\mathrm{L} \circ \mathrm{t} s$} & \multicolumn{2}{|c|}{$\begin{array}{l}\text { Métacercaires } \\
\text { admın1s trées }\end{array}$} & \multirow{2}{*}{$\begin{array}{c}\text { Examens } \\
\text { coproscoprques }\end{array}$} & \multirow{2}{*}{$\begin{array}{c}\text { Durée de } \\
\text { l'expérience }\end{array}$} & \multirow{2}{*}{ Résultats des autopsies } \\
\hline & Nombre & Age & & & \\
\hline $\begin{array}{l}\text { Lot } n^{\circ} \mathrm{I} \\
\text { Lapin } n^{\circ} 1 \\
\text { Lapin } n^{\circ} 2 \\
\text { Lapin } n^{\circ} 3 \\
\text { Lapin } n^{\circ} 4\end{array}$ & $\begin{array}{l}60 \\
58 \\
30 \\
30\end{array}$ & $\begin{array}{c}18 \text { jours } \\
" \\
" \\
"\end{array}$ & $\begin{array}{c}\text { Tous négatifs } \\
" \\
" \\
"\end{array}$ & $\begin{array}{l}85 \text { jours } \\
"- \\
"- \\
11\end{array}$ & $\begin{array}{l}\text { Traces d'Angiocholite sur l'un des } \\
\text { lobes du foie - Pas de Easciola } \\
\text { Foie, poumon et vésicule biliarre } \\
\text { en bon état et sans parasites } \\
\text { - idem - } \\
\text { - idem - }\end{array}$ \\
\hline $\begin{array}{l}\text { Lot } n^{\circ} \mathrm{II} \\
\text { Lapin } n^{\circ} 5 \\
\text { Lapin } n^{\circ} 6^{+} \\
\text {Lapin } n^{\circ} 7^{+} \\
\text {Lapin } n^{\circ} 8\end{array}$ & $\begin{array}{l}30 \\
30 \\
60\end{array}$ & $\begin{array}{c}64 \text { jours } \\
\text { " } \\
" 1\end{array}$ & " & $\begin{array}{r}101 \text { jours } \\
89 \text { jours } \\
65 \text { jours } \\
101 \text { jours }\end{array}$ & $\begin{array}{l}\text { - idem - } \\
\text { - idem - } \\
\text { Traces d'Angiocholite - Quelques } \\
\text { nodules partiellement calcufiés } \\
\text { Pas de parasites. } \\
\text { Fole en mauvais état.Deux gros abcès } \\
\text { en voie de calcification-I } \bar{F} \cdot g^{i} \text { gantica }\end{array}$ \\
\hline $\begin{array}{l}\text { Lot } n^{\circ} \text { III } \\
\text { Lapin } n^{\circ} 9 \\
\text { Lapin } n^{\circ} 10 \\
\text { Lapin } n^{\circ} 11 \\
\text { Lapin } n^{\circ} 12\end{array}$ & $\begin{array}{l}200 \\
200 \\
200 \\
200\end{array}$ & $\begin{array}{c}158 \text { jours } \\
" \\
" \\
"\end{array}$ & $\begin{array}{l}- \\
- \\
-\end{array}$ & $\begin{array}{c}57 \text { jours } \\
" \\
" \\
"\end{array}$ & $\begin{aligned} \text { Foie en bon état - Pas de parasites } \\
\text { - idem - } \\
\text { - idem - } \\
\text { - idem - }\end{aligned}$ \\
\hline
\end{tabular}

+ mort naturelle.

hépatiques provoquées - semble-t-il - par le parasite au cours de sa migration dans le foie.

Chez les lapins 1 et 7 , on observe une inflammation des voies biliaires peu marquée et limitée à l'un des lobes. Cette cholangite ne touche que quelques canaux qui prennent l'aspect de bandes ou de traînées grisâtres, irrégulières, s'enfonçant dans le parenchyme hépatique.

Chez les lapins 7 et 8, les lésions d'angiocholite s'accompagnent d'une augmentation de volume de l'organe dans l'épaisseur duquel sont inclus des nodules remplis d'une substance nécrotique jaune en cours de calcification.

Chez le lapin $\mathrm{n}^{\circ} 8$, on a affaire à de véritables abcès, également en voie de calcification. Le foie est en mauvais état, dur, scléreux, adhérent aux organes voisins et recouvert de dépôts de fibrine.

Après extraction par la méthode de Yalcin (34), une seule Fasciola gigantica a pu être isolée dans le foie du lapin $\mathrm{n}^{\circ} 8$, sacrifié 101 jours après avoir reçu 58 Métacercaires de 9 semaines. Elle mesure $19 \mathrm{~mm}$ de long sur 4,2 $\mathrm{mm}$ de large. L'utérus renferme quelques cuufs qui ont été retrouvés dans la bile (vésicule), mais non dans les selles.

\section{COMMENTAIRES}

1. Il ressort de cette étude que le lapin semble relativement résistant à l'infestation par Fasciola gigantica et que, dans cette espèce, les Douves se développent $\mathrm{mal}$ et en nombre restreint.

Cette opinion est partagée par COYLE (5), DAVTYAN (10), GURALP et Collab. (16) et par SEWELL (29). THAPAR et TANDON (31) ne réussissent pas à transmettre la maladie à un lapin, bien que le nombre de métacercaires administrées soit élevé (252).

Plus récemment, MANGO et Collab. (24) au Kenya, comparant le pouvoir infestant des Métacercaires de Fasciola gigantica sur plusieurs animaux de laboratoire, arrivent à des conclusions voisines : les espèces les plus réceptives sont le hamster, le cobaye et la souris. Le lapin ne vient que bien après et les Fasciola 
mises en évidence 22 semaines après l'infestation initiale $\left({ }^{*}\right)$ sont toujours immatures.

De même, SAHBA et Collab. (32), en Iran, ne parviennent à infester qu'un seul lapin sur cinq. Ils obtiennent, à l'autopsie une dizaine de semaines plus tard, des parasites mesurant en moyenne $15,8 \times 4,2 \mathrm{~mm}$.

Il n'en est pas toujours ainsi et d'autres expériences ont donné de meilleurs résultats :

- Annie PORTER (26), en Afrique du Sud, nourrit un lapin avec des feuilles de salade sur lesquelles des Métacercaires de Fasciola gigantica sont enkystées. 64 jours plus tard, l'animal meurt dans un état d'extrême maigreur et 20 Douves adultes sont découvertes dans le foie.

- FOUAD ABDEL GHANI (13), en Egypte, utilise cinq lapins et chacun d'eux reçoit 10 Métacercaires d'une semaine. L'auteur tue les animaux au bout de $30,75,84$ et 90 jours. Il constate que trois d'entre eux ont réagi favorablement et hébergent des Distomes. Les Douves mesurent $1,30 \mathrm{~mm} \times 400 \mu$ à 30 jours, $10,5 \times 3 \mathrm{~mm}$ à 75 jours et $13 \times$ $2,76 \mathrm{~mm}$ à 84 jours. Elles sont toutes immatures, mais les organes génitaux sont déjà bien formés.

- KADHIM et ALTAIF (20), en Iraq, distribuent à des lapins 50 à 200 Métacercaires d'une semaine. Des œufs de Fasciola gigantica sont éliminés dans les selles au bout de 8790 jours, ce qui confirme, à peu de choses près, les essais d'ALICATA (2) aux îles Hawaii.

2. De telles différences dans le comportement du lapin sont difficilement explicables.

2.1. MANGO et Collab. (24) suggèrent l'existence, en Afrique de l'Est, d'une souche de Fasciola gigantica qui serait moins infestante pour le lapin que celles d'Afrique du Sud, des îles Hawaii, du Proche Orient ou d'Egypte. $\mathrm{Si}$ cette hypothèse est exacte, la souche du Tchad se rapprocherait de celle du Kenya.

2.2. D'autres facteurs doivent également être pris en considération, notamment le nombre de Métacercaires administrées. A l'exclusion des essais de FOUAD ABDEL GHANI (13), les infestations réussies l'ont été à partir

(*) 10 à 15 Métacercaires par animal. de 100 Métacercaires. KADHIM et ALTAIF (20) insistent sur ce point: plus le nombre de Métacercaires augmente et plus les infestations risquent d'être positives. L'ingestion par le lapin de 100-200 Métacercaires favorise l'apparition d'une distomatose chronique avec élimination régulière d'œufs dans un délai de 90 jours. SRIVASTATA et SINGH (30) vont plus loin. Selon eux, il faut environ 1000 Métacercaires pour déclencher, 63 jours après, une distomatose aiguë accompagnée d'importantes lésions du parenchyme hépatique et des canaux biliaires, lésions que les auteurs décrivent avec précision. Des modifications de cette ampleur ne sont d'ailleurs pas constantes et, dans cette expérience, ne concernaient qu'un seul des treize lapins infestés.

Le rendement Douves/Métacercaires paraît faible : 4 à 10 p. $100(24) ; 10$ p. 100 (13). Même dans le cas d'infestations massives, les Douves sont peu nombreuses dans le parenchyme hépatique : 4 exemplaires dans la moitié du foie examiné par SRIVASTATA et SINGH (30).

Par comparaison, l'infestation du lapin par des Métacercaires de Fasciola hepatica est beaucoup plus facile, comme le prouvent les recherches d'HUGHES (19) qui donne 15 à 50 Métacercaires par animal. Les premiers cufs apparaissent au bout de 51-54 jours. A l'autopsie, on trouve, en moyenne 5-9 Fasciola pour 15 Métacercaires et 5-36 parasites pour 50. Le rendement Douves/Métacercaires, dans le premier cas, est de 63 p. 100 et de 48 p. 100 , dans le second.

2.3. Les Métacercaires de Fasciola gigantica, pour être pleinement infestantes, doivent subir un temps de maturation d'au moins une semaine (13).

Dans toutes les expériences dont il vient d'être question, ce délai a été respecté et l'on ne peut arguer de ce fait pour expliquer les différences observées.

3. Dans la nature, la présence de Fasciola gigantica semble, dans l'état actuel de nos connaissances, exceptionnelle chez les Léporidés africains, alors que les Léporidés européens sont souvent atteints de distomatose à Fasciola hepatica.

En Egypte, deux cas ont été rapportés par ABDOU (1) et par EZZAT et ABDEL GHA- 


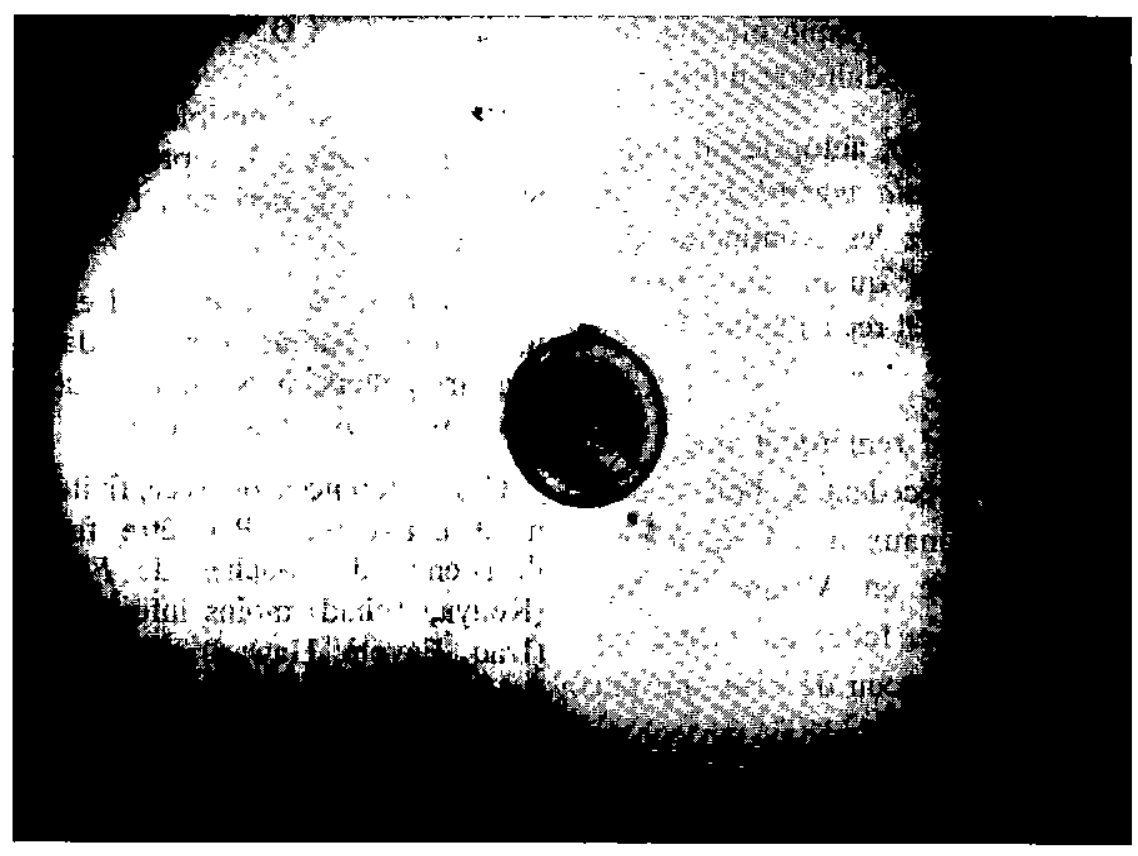

Photo 1. - Métacercaire de Fasciola gigantica avec ses glandes de pénétration.

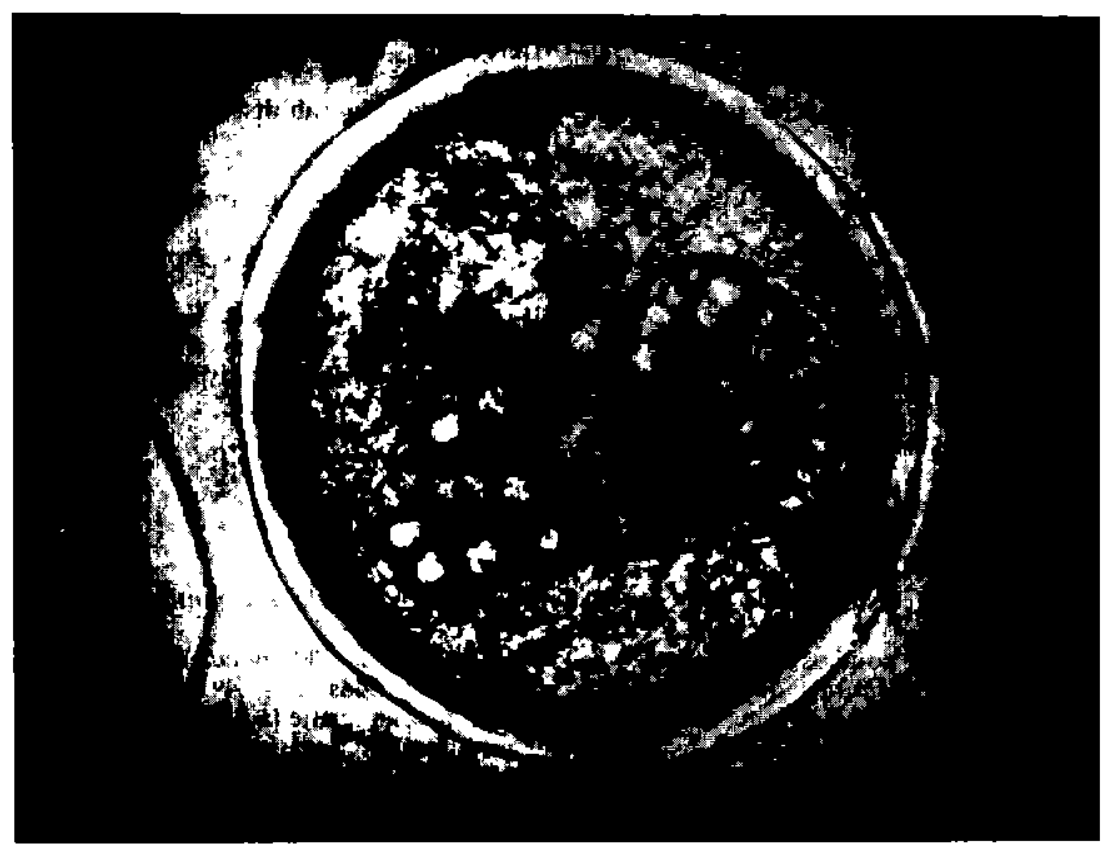

Photo 2. - Métacercaire de Fasciola gigantica. Détails. 
NI (12) qui, dans le foie d'un lapin malade, ont isolé six Fasciola gigantica adultes et mûres.

En Uganda, COYLE (6), autopsie 50 lapins dans une zone où Fasciola gigantica est fréquemment recontrée chez les Ruminants domestiques: il ne trouve qu'un seul animal atteint dans le foie duquel il recueille une Douve de $10 \mathrm{~mm}$.

HAMMOND (17) fait remarquer que, hormis les observations précédentes, l'infestation naturelle des petits animaux n'a, jusqu'à présent, jamais été signalée en Afrique, ce que confirment des recherches faites au Tchad sur les parasites du lièvre : aucun de ceux qui ont été examinés de 1954 à 1969 n'était porteur de Fasciola gigantica, alors que, dans ce pays, le taux moyen d'infestation du zébu est de 30 p. 100.

En tout état de cause, il est difficile actuellement de tirer des conclusions définitives, les renseignements dont on dispose étant trop fragmentaires et trop peu nombreux.

\section{CONCLUSIONS}

Le lapin est sensible à l'infestation par Fasciola hepatica. Il représente, de ce fait, un bon animal d'expérience, très utilisé au laboratoire.

Avec Fasciola gigantica, il en va tout autrement. Les résultats obtenus dans divers pays sont irréguliers, nuls ou presque dans certains cas, excellents dans d'autres.

Ces différences de réceptivité sont difficilement explicables. Peut-être faut-il incriminer l'existence de souches de Fasciola gigantica (Kenya, Tchad) moins infestantes que d'autres (Iraq, Egypte, Hawaii) ou encore l'administration au lapin d'un trop petit nombre de Métacercaires.

\section{Remerciements}

L'auteur tient à remercier vivement Monsieur le Professeur EUZEBY pour les facilités accordées dans son Laboratoire et Messieurs TRONCY et BIRGI qui se sont chargés de la récolte et de l'expédition des Limnées et des œufs de Fasciola gigantica.

\section{SUMMARY}

\section{Fascioliasis with Fasciola gigantica in rabbits}

Twelve french rabbits received 30 to 200 Metacercariae of Fasciola gigantica each. The cysts were 18,64 and 158 days old and stored at $+4^{\circ} \mathrm{C}$.

Three animals were apparently infected and one fluke only was recovered in the liver.

The author compares the result with these obtained in another countries. The rabbit seems to be irregulary susceptible to the experimental infection with Fasciola gigantica. The observed disparity may be related to strains used and number of cysts administered.

\section{RESUMEN}

\section{La distomatosis del conejo doméstico con Fasciola gigantica}

Doce conejos franceses reciben cada uno 30 a 200 metacercarias de Fasciola gigantica de 18,64 y 158 días, conservadas a $+4^{\circ} \mathrm{C}$.

Tres animales reaccionan positivamente a la enfermedad experimental, pero se recoge una sola distoma en el parenquima hepatico.

EI autor compara estos resultados a los obtenidos en otros paises.

Comproba que el conejo reacciona irregularmente a la infestación con Fasciola gigantica y que su receptividad varia en función de las cepas utilizadas y del número de metacercarias administrados. 


\section{BIBLIOGRAPHIE}

1. ABDOU (A. H.). Fasciola gigantica infection in an egyptian rabbit. J. Arab. vet. med. Ass., 1961, $21(1 / 2)$ : 41-43.

2. ALICATA (J.E.). Observations on the life-history of Fasciola gigantica, the common liver fluke of cattle in Hawaii and the intermediate host Fossaria ollula. Hawaii Agric. exp. Station, Bull., $1938(80): 22$.

3. BITAKARAMIRE (P. K.). Lymnaea natalensis laboratory culture and production of Fasciola gigantica metacercariae. Parasitology, 1968, 58 : 653-656.

4. BORAY (J. C.). Experimental fascioliasis in Australia Adv. Parasit., 1969, 7: 96-204

5. COYLE (T. J.). Control of fascioliasis in Uganda. I.A.C.E.D. Symposium on Helminthiasis in domestic animais, Nairobi, 1959.

6. COYLE (T. J.). The epidemiology of Fasciola gigantica in cattle in Uganda protectorate. Thesis, Royal College of Veterinary surgeons, 1961.

7. DARGIE (J.D.), HOLMES (P. H.), Mc LEAN (J.M.) et MULLIGAN (W.). Further studies on the anaemia in fascioliasis; simultaneous use of $51_{\mathrm{Cr}}$ labelled red cells and $95_{\mathrm{Nb}}$ labelled albumin. Vet. Rec., 1968, 82 (12): 360-361.

8. DARGIE (J. D.), HOLMES (P. H.), Mc LEAN (J. M.) et MULLIGAN (W.). Pathophysiology of fascioliasis in the rabbit. Studies on albumin turnover. J. comp. Path. Ther., 1968, 78 (1): $101-105$.

9. DARGIE (J.D.) et MULLIGAN (W.). The onset and development of anaemia and hypoalbuminaemia in rabbits infected with Fasciola hepatica. J. comp. Path. Ther., 1971, 81 (2): 187-202.

10. DAVTYAN (E. A.). Pathology of different species of Fasciola and its variability depending on the developmental conditions. Zool. Zh., 1956, 35 (11): $1617-1625$ (en russe).

11. EUZEBY (j.). Les maladies vermineuses des animaux domestiques et leurs incidences sur la pathologie humaine. T. II. Maladies dues aux Plathelminthes, Fasc. 2, Liv. I. Paris, Vigot frères, 1971.

12. EZZAT (M. A. E.) et FOUAD ABDEL GHANI (A.). The daily output of Fasciola gigantica eggs as estimated from a naturally infected rabbit. Proc. Ith. Ann. vet. med. Cong., Le Caire, 1962, 341-347.

13. FOUAD ABDEL GHANI (A.). Experimental infection of animals with Fasciola and Paramphistomum cysts. Agric. Res. Rev., Cairo, 1960, 38 (2): 375-387.

14. GIGITASHIVILI (M.S.). Experimental fascioliasis in rabbits. Trudy nauchno-issled. Inst. med. Parazyt. trop. Med., 1969, 4: 23-27 (en russe).

15. GOLDBERGIENNE (M.). Study on the biochemical indicators in the blood of rabbits experimentally infected with Fasciola. Acta parasit. Lith., 1962, 4: 55-64.

16. GURALP (N.), OZCAN (C.) et SIMMS (B. T.). Fasciola gigantica and fascioliasis in Turkey. Am. J. vet. Res., 1964, 25 (104) : 196-210.

17. HAMMOND (J. A.). Infection with Fasciola Sp. in wildlife in Africa. Trop. anim. Hlth. Prod., 1972, 4 (1) : 1-13.
18. HOLMES (P. H.), DARGIE (J. D.), Mc LEAN (J.M.) et MULLIGAN (W.). The anaemia in fascioliasis studies with $51 \mathrm{Cr}$ labelled red cells, J. comp. Path. Ther., 1968, 78 (4) : 415-420.

19. HUGHES (D. L.). M. Sc. Thesis, University of London, 1959. In BEN DAWES et HUGHES (D. L.). Fascioliasis : the invasive stages of Fasciola hepatica in mammalian hosts. Adv. Parasit., 1964, 2 : 105.

20. KADHIM (J.K.) et ALTAIF (K. I.). The experimental demonstration of Lymnaea lagoris euphratica as an intermediate host of Fasciola gigantica in Iraq. Ann. trop. Med. Parasit., 1970, 64 (3): 335-337.

21. KENDALL (S. B.) et SINCLAIR (I. J.). Barriers to reinfection with Fasciola hepatica in the rabbit. Res. vet. Sci., 1971, 12 (1) : 74-79.

22. KIMURA (S.). Experimental studies on fascioliasis. I. Infection rate of metacercariae in rabbits, distribution of Fasciola hepatica in the liver and relation between the number of parasites and death of infected rabbits. Jap. J. Parasit., 1961, $10(1): 45-51$.

23. KIMURA (S.). Experimental studies on fascioliasis. II. Clinical and haematological observations in infected rabbits. Jap. J. Parasit., 1961, 10 (3): 536-541.

24. MANGO (A. M.), MANGO (C. K. A.) et ESAMAL (D.). A preliminary note on the susceptibility, prepatency and recovery of Fasciola gigantica in small laboratory animals. J. Helminth., 1972, 46 (4) : 381-386.

25. MOVSESIJAN (M.), LALIC (R.) et BOROJEVIC (D.). The use of radioactive $51 \mathrm{Cr}$ to investigate anaemia in rats and rabbits infected with Fasciola hepatica. Vet. Glasn., 1970, 2 (4) : 343-347.

26. PORTER (A.). The life-history of the african sheep and cattle fluke, Fasciola gigantica. S. Afr. J. Sci., 1920, $17: 126-130$.

27. ROSS (J. G.). Studies of immunity to Fasciola hepatica: acquired immunity in cattle, sheep and rabbits following natural infection and vaccine procedures. J. Helminth., 1967, 41 (4) : 393-399.

28. SAHBA (G. H.), ARFAA (F.), FARAMANDIAN (I.) et JALALI (H.). Animal fascioliasis in Khuzestan, Southwestern Iran. Parasitology, 1972, 58 (4) : 712-716.

29. SEWELL (M.M.H.). The pathogenesis of fascioliasis. Vet. Rec., 1966, 78 (3) : 98-105.

30. SRIVASTATA (P.S.) et SINGH (K.S.). Some observations on the pathology of experimental Fasciola gigantica infection in rabbits. Indian $J$. anim. Sci., 1972, 42 (1): 72-76.

31. THAPAR (G.S.) et TANDON (R.S.). On the life-history of liver fluke Fasciola gigantica Cobbold, 1855 in India. Indian J. Helminth., 1952, 4 (2) : 77-112.

32. URQUARTH (G.M.). The rabbit as host in experimental fascioliasis. Expl. Parasit., 1954, 3: 38-44.

33. URQUARTH (G. M.). The pathology of experimental fascioliasis in the rabbit. J. Path. Bact., 1956, 71 : 301-310.

34. YALCIN (M.). Récolte des grandes Douves adultes et immatures. C.R. Journées Parasit. Avidila-E.N.V. Alfort, 1970: 80-1. 